\title{
Three-Dimensional Computed Tomography (3-D CT) for Evaluation and Management of Children with Complex Chest Wall Anomalies: Useful Information or Just Pretty Pictures?
}

\author{
E. Hollin Calloway, B.A. ${ }^{1}$, Ali N. Chhotani, M.D. ${ }^{1}$, Yueh Z. Lee, M.D./Ph.D. ${ }^{2}$, and J. Duncan \\ Phillips, M.D. ${ }^{1}$ \\ ${ }^{1}$ Department of Surgery, UNC Chapel Hill School of Medicine, University of North Carolina at \\ Chapel Hill, Chapel Hill, NC, USA \\ 2Department of Radiology, UNC Chapel Hill School of Medicine, University of North Carolina at \\ Chapel Hill, Chapel Hill, NC, USA
}

\section{Abstract}

\begin{abstract}
Purpose-Shaded Surface Display (SSD) technology, with 3-D CT reconstruction, has been reported in a few small series of patients with congenital or acquired chest wall deformities. SSD images are visually attractive and educational, but many institutions are hesitant to utilize these secondary to cost and image data storage concerns. This study was designed to assess the true value of SSD to the patient, family, and operating surgeon, in the evaluation and management of these children.
\end{abstract}

\begin{abstract}
Methods-Following IRB approval, we performed a retrospective review of records of 82 patients with chest wall deformities, evaluated with SSD, from 2002 to 2009. SSD usefulness, when compared with routine 2-D CT, was graded on a strict numerical scale from 0 (added no value besides education for the patient/family) to 3 (critical for surgical planning and patient management).
\end{abstract}

Results-There were 56 males and 26 females. Median age was 15.3 years (range: 0.6-41.1). Deformities included 56 pectus excavatum, 19 pectus carinatum, and 8 other/mixed deformities. 6 patients also had acquired asphyxiating thoracic dystrophy (AATD). Eleven (13\%) had previous chest wall reconstructive surgery. In $25(30 \%)$ patients, SSD was useful or critical. Findings underappreciated on 2-D images included: sternal abnormalities (29), rib abnormalities (28), and heterotopic calcifications (7). SSD changed or influenced operation choice (4), clarified bone versus soft tissue (3), helped clarify AATD (3), and aided in rib graft evaluation (2). Point biserial correlation coefficient analysis $\left(\mathrm{R}_{\mathrm{pb}}\right)$ displayed significance for SSD usefulness in patients with previous chest repair surgery $\left(\mathrm{R}_{\mathrm{pb}}=0.48, \mathrm{p} \unlhd .001\right)$, AATD $\left(\mathrm{R}_{\mathrm{pb}}=0.34, \mathrm{p}=0.001\right)$, pectus carinatum $\left(\mathrm{R}_{\mathrm{pb}}=0.27, \mathrm{p}=0.008\right)$, and females $\left(\mathrm{R}_{\mathrm{pb}}=0.19, \mathrm{p}=0.044\right)$.

Conclusions-Shaded Surface Display, when used to evaluate children and young adults with congenital or acquired chest wall deformities, provides useful or critical information for surgical

\footnotetext{
(C) 2010 Elsevier Inc. All rights reserved.

Corresponding Author: E. Hollin Calloway, Elizabeth_calloway@med.unc.edu, Telephone: 919-368-8009, Fax: 919-350-7859, Address: Hollin Calloway, 318 McCauley St \#1, Chapel Hill, NC 27516.

Publisher's Disclaimer: This is a PDF file of an unedited manuscript that has been accepted for publication. As a service to our customers we are providing this early version of the manuscript. The manuscript will undergo copyediting, typesetting, and review of the resulting proof before it is published in its final citable form. Please note that during the production process errors may be discovered which could affect the content, and all legal disclaimers that apply to the journal pertain.
} 
planning and patient management in almost $1 / 3$ of patients, especially those requiring a second operation, with acquired asphyxiating thoracic dystrophy, pectus carinatum, and females.

\section{Keywords}

pectus excavatum; pectus carinatum; computed tomography (CT); shaded surface display (SSD); acquired asphyxiating thoracic dystrophy (AATD)

\section{INTRODUCTION}

Pectus excavatum (PE), pectus carinatum (PC), acquired asphyxiating thoracic dystrophy (AATD)(1-3), and other pediatric chest wall deformities may present in multiple varied configurations. Surgical repair of some chest wall deformities may be successfully accomplished with the minimally invasive (i.e. "Nuss") technique $(4,5)$, or one of its modifications(6). Other patients may be best served using more traditional "open" (i.e. "Ravitch") techniques(7-9). Yet other complex patients may require more creative surgical approaches(10), including sternotomy with rib grafting(11). Therefore, it is important for the pediatric surgeon to have access to the best possible pre-operative evaluation of a patient's particular condition before choosing the correct surgical procedure and tailoring it to the individual deformity.

Computed tomography (CT) has been advocated as the best preoperative imaging test for evaluation of these patients (12-17). For PE, the pectus index (or Haller index), a ratio of the measured inside width of the chest divided by the narrowest anterior-posterior diameter of sternum to spine, is commonly used to assess severity $(18,19)$. Preoperative CT has also been found to frequently show additional abnormalities (12), allow calculation of a cardiac deformity index (13), improve Nuss bar measurement and design (16), and allow for objective measurements of postoperative cardiac decompression (15). For PC, pre-treatment CT has been shown to guide compressive orthotic bracing $(14,17)$.

Shaded Surface Display (SSD) 3-D reconstruction technology converts 2-D CT scans into rotatable, 3-D renderings of calcified tissue (bones). Use of SSD with 3-dimensional reformatting of CT scan images has been reported in only a few small series of pediatric patients with pectus deformities $(20,21)$. Although these reports suggested that 3-D reconstructions improved surgeons' abilities to determine exact angles of the ribs and costal cartilage to the sternum, and thus enhanced individual operative planning, the reports have been primarily limited to presentation of attractive pictures, which are interesting and potentially educational to families and patients $(20,21)$. Critical analysis of this technology's precise usefulness for assessment of patients with complex chest wall anomalies has been lacking.

We designed a substantially larger study to specifically determine the subset of patients for which the use of SSD could be more informative for surgeons. We hypothesized that 3-D reconstructions of CT scan images obtained by SSD technology would enhance a surgeon's ability to better plan operations, evaluate, and manage a subset of patients with complex chest wall anomalies. Additionally, because surgeons may encounter unexpected intraoperative findings during chest repair, preoperative analysis with SSD technology could forewarn surgeons of specific problems and deformities, which may not have been clear from 2-D CT scans. We aimed to specifically define the subset of patients, in terms of deformity characteristics and anomalies discovered pre-operatively, which would benefit most from the use of SSD. 


\section{METHODS}

Following IRB approval (protocol \# 07-0538 UNC-Chapel Hill), we performed a retrospective review of records of 82 patients (mean age 15.3 years, range 0.56 to 41.1 years) with chest wall deformities, who were evaluated with both 2-D CT and SSD, from 2002 to 2009. Fifty-six (68\%) were male and 26 (32\%) were female. Approximately $75 \%$ of chest wall patients evaluated during this time period at our institution had these studies performed. The decision on whether to request a 3-dimensional $\mathrm{CT}$ preoperatively was at the discretion of the evaluating surgeon.

CT images were obtained on the SOMATOM Sensation 40 multislice CT scanner, using Somaris 5.1 software. Radiation dose for all studies was done according to ALARA (as low as reasonably achievable) methods. SSD images were created on the Multimodality Workplace software (Siemens Medical Solutions USA, Inc., Malvern, PA). 3-D reconstructions were derived from 2-D data, with no additional radiation dose delivered to the patients.

Although radiographic images were initially interpreted by fellowship-trained pediatric radiologists, for this study the actual images were evaluated by one of three fellowshiptrained, board-certified pediatric surgeons. Each had at least ten years of previous experience with pediatric chest wall surgery and CT evaluation. No attempt was made, during the study period, to standardize the preoperative evaluation protocol.

SSD usefulness, for chest wall assessment and surgical planning, when compared with routine 2-D CT, was graded by the surgeons on a strict numerical scale that ranged from 0 to 3 defined as follows:

(0) SSD added no value besides preoperative education to the patient and family;

(1) SSD provided insight into the patient's deformities but did not add value to the 2-D CT, for the specific operation, evaluation, or management of the patient;

(2) SSD added important insight into the patient's deformity that was unclear in the 2-D scan;

(3) SSD was critical for operative planning or patient evaluation and management;

Patients were divided into groups based on their primary deformities. Patients assigned a 0 or 1 from the above mentioned scale were deemed "SSD not superior to 2-D CT scan alone", and patients with a 2 or 3 were deemed "SSD useful and necessary for evaluation and management", creating a binary ranking. Numerical results were analyzed using point biserial correlation coefficient analysis, correlating usefulness of SSD in the different deformity types, age, gender, and presence or absence of previous chest wall repair (R. Paul Thomlinson, Ph.D., Springfield, MO). P-values $\leq 0.05$ were considered statistically significant.

\section{RESULTS}

Of the 82 patients evaluated, patient deformities included 53 PE only, 16 PC only, 6 AATD (all requiring a second operation), $5 \mathrm{PE}$ and PC mix, 1 Poland's Syndrome (PS), and 1 PS and PC mix (Table 1). Eleven of $82(13 \%)$ had undergone previous chest wall reconstructive surgery.

In 25 of 82 cases (30\%), the value assigned to the 3-D images was either a 2 or a 3, implying that SSD imaging was either very useful or critical for surgical planning or patient management (see Table 2). Findings poorly appreciated on 2-D but that were clearly 
delineated with 3-D are described below and listed in Table 2. Most patients had more than one finding.

There were 29 patients (35\%) with sternal abnormalities such as abnormal segmentation, effects from previous surgery, and ultra-short length (see Figure 1). Sternal abnormalities also included rotation (twist around the vertical axis) as in Figure 2, and angulation (leftward or rightward tilt if viewed from coronal plane).

Inward bony rib curvature (rather than just cartilage angulation/curvature) shown in Figure 2 , or outward rib protrusion, was found in 20 patients (24\%). Rib abnormalities such as fused, bifid, or missing ribs (Figure 3) were found in 8 patients (10\%).

SSD provided a clearer differentiation between calcified bone and soft tissue in 3 patients $(4 \%)$. See Figures 2 and 4.

Heterotopic calcifications were better clarified in 7 patients (9\%). See Figures 5 and 6.

There were 4 cases (5\%) where SSD changed or highly influenced a surgeon's decision to choose a particular operation. See Figure 2.

In 3 patients (4\%), SSD provided a clearer view of AATD than seen on the 2-D slices, as in Figure 5.

In 2 patients (2\%), SSD clarified whether or not rib grafts (which had been placed in a previous operation) were calcifying or not.

Statistical analysis by point biserial correlation coefficients (Table 3 ) showed significance for SSD usefulness in patients with previous chest repair surgery as in $\left(\mathrm{R}_{\mathrm{pb}}=0.48, \mathrm{p} \unlhd .001\right)$ (as in Figures 4, 5, 6 and 7), AATD $\left(\mathrm{R}_{\mathrm{pb}}=0.34, \mathrm{p}=0.001\right)$ (as in Figures 5 and 7), pectus carinatum $\left(\mathrm{R}_{\mathrm{pb}}=0.27, \mathrm{p}=0.008\right)$ (as in Figures 3 and 8), and females $\left(\mathrm{R}_{\mathrm{pb}}=0.19, \mathrm{p}=0.044\right)$.

\section{DISCUSSION}

To date, only two small series have examined the usefulness of Shaded Surface Display in chest wall reconstruction evaluation and planning. Pretorius et al. evaluated eight patients who required a second operation and suggested that 3-D reconstruction technology was useful in operative planning and improved outcomes in patients with complex chest wall deformities(21). Albes et al. presented a case review of two patients with complex pectus carinatum and pectus excavatum deformities, and concluded that operative management could be substantially improved by assessing 3-D reconstructions of 2-D CT scans preoperatively(20). Our study directly compared findings of 2-D and 3-D in 82 patients and focused on identifying the specific characteristics of patients that would lead a pediatric surgeon to utilize the 3-D reconstruction for assessment, beyond just patients undergoing second operations.

Sternal size, shape, and configuration may influence surgical planning. For example, the presence of an ultra-short sternum might make it difficult for the surgeon to achieve adequate anterior chest wall protrusion with placement of a Nuss bar. Severe sternal rotation might cause the sternum to lie at an angle that is too close to perpendicular to the bar(s), and is considered by some, to be a relative contraindication to the Nuss procedure. Although sternal length and rotation can be assessed to some degree by a careful preoperative physical examination, a boney sternal defect or an unfused sternum may not and might be too weak to support the pressure of a Nuss bar(s). These findings are clarified by SSD and important 
for the surgeon's awareness, and likely would modify the procedure or suggest the need for a Ravitch procedure.

SSD is excellent at clarifying bone from soft tissue. Although 3-D CT imaging reformatting software can be adjusted to demonstrate soft tissue, including blood vessels and solid organs, SSD is typically used and set up on most systems to not render images of soft tissue. Thus, by comparing SSD to 2-D CT, the presence of soft tissue can be inferred. Identification of heterotopic calcifications versus soft tissue enables a surgeon to plan for multiple Nuss bars in the location of that tissue when needed, since calcified tissue typically requires much more force for elevation. In our experience, most PE patients needing a second operation have significant heterotopic calcifications.

Since multiple costal cartilages are typically resected in a Ravitch procedure, preoperative identification of the specific location of a fused, missing, or bifid rib cartilage, and its intersection with the sternum, helped plan and alter the proposed operation. In addition, since the tips of severely deformed inward-curving boney ribs can be resected in the subperiosteal plane with the Ravitch procedure, but not with the Nuss, inward boney rib curvature was an important radiographic finding. SSD provides a much clearer view of how deeply the tips protrude than 2-D CT reveals.

In 4 cases, the SSD view alone changed the surgeon's decision about the operative procedure he had decided upon by physical exam, plain film, and 2-D CT. The first case was a 41 year old female with recurrent PE who had a previous Ravitch repair at age 3. 2-D CT revealed significant heterotopic calcifications, sternal depression, and ribs that had healed into her chest. From 2-D CT alone a Nuss procedure would have been indicated. But after analyzing the SSD, which clarified the extent of inward rib curvature, the surgeon decided that a Ravitch procedure would be more appropriate because portions of ribs needed to be resected.

The second patient was a 12 year old female with a marked cosmetic protuberant rib anomaly and significant chest pain. The initial assessment based on physical exam was that her pain was likely due to PC and the plan was to perform a modified Ravitch procedure to resect some of the protruding cartilages. 2-D CT showed that the patient actually did not have a significant pectus deformity (only mild PC), and that surgery was unlikely to be indicated. 3-D reconstruction of this patient's complex sternal anatomy further clarified the specific rib deformity (missing left ribs 3 and 11, and right ribs 1, 3, 10, and 12), but also showed a normal intrathoracic volume and contour, which further confirmed to the surgeon that surgery was not indicated. Instead other tests were recommended to discover the source of her chest pain, as she had a history of asthma and possible costochondritis.

The third patient was a 21 year old female with recurrent PE after a Nuss repair at age 12, after which the Nuss bars slipped. SSD clarified the extent of sternal rotation, which was visible on 2-D CT. However, the volumetric rendering of the 3-D clarified the angulation and provided a much better view of the extensive heterotopic calcifications and calcified costal cartilages due to previous Nuss bar slippage. SSD revealed that a Ravitch rather than a Nuss procedure was indicated. The fourth patient was the male with PC in Figure 2 with inward protruding ribs that required resection. SSD influenced the surgeon to perform a Ravitch rather than a Nuss procedure.

Finally, the ability to visualize remnant scars or bone grafts from previous operations was enhanced on SSD relative to 2-D CT. For example, this enabled the surgeon to better manage a patient with grafted ribs or cartilaginous tissue and note the progress of calcification. Scars or remnants from a previous operation, in more complex cases such as the male in Figure 5, are better viewed in 3-D, clarifying the exact location of a previous 
sternal osteotomy. This influenced the surgeon to use 3 instead of 2 Nuss bars for the child's second operation. There were two patients who had rib grafts who scored a 2 or 3 on the 3-D value scale, indicating usefulness of SSD. These two patients both had acquired Jeune's syndrome due to previous operations, and required extensive remodeling including grafts (a sternal-split procedure, where rib grafts are placed between two segments of sternum, which has been split) to open up their tiny chest cavities in their second operations. The SSD postoperatively enabled the surgeon to visualize whether the grafts had begun to calcify, an indication that they were mineralizing and working instead of failing. These examples may be the best demonstration of the merits of SSD in these complex patients.

This study appears to define a subset of patients who will most benefit from evaluation with 3-D reconstruction. Since SSD software packages are now routinely included at no extra cost with the purchase of new CT scanners, pediatric surgeons who perform chest wall reconstruction procedures may consider routinely utilizing this technology in selected patients. There is no additional radiation exposure to the patient with these 3-D imaging techniques. Since most health insurance companies do not provide additional financial reimbursement for radiographic interpretation charges, our patients receive no additional billing for 3-D image interpretations. It typically takes the technologist less than five minutes to perform the reconstructions, including transferring the data to the central storage system.

The only potential additional financial cost of SSD may be the extra storage space of imagegenerated data. However, since the total amount of increased storage for the image data of each patient undergoing 3-D reconstructions is roughly $22 \mathrm{MB}$ (megabytes), and since the rough cost of storage is $\$ 100$ per terabyte, approximately 50,000 patients worth of data could be captured in one terabyte, at a cost to the institution of only $\$ 100$ per year. However, by determining this subset of patients who benefit from 3-D data, storage resources can be optimized, especially in a healthcare facility that may have a tighter data- storage budget.

The main limitation of this study is that it was done in a retrospective fashion. Thus, one could argue that results were biased, since outcomes of the majority of the surgeries were known before the study began. However, it might also be argued that, for a study such as this one, a retrospective review might be the best since intraoperative findings (chest wall stiffness, location of calcifications, etc.) could be assessed and correlated with the preoperative CT images.

Shaded Surface Display provided useful or critical information for surgical planning and patient management in almost one third of our patients, and was especially important for (i) patients requiring a second operation, (ii) those with acquired asphyxiating thoracic dystrophy, (iii) those with pectus carinatum, and (iv) in females. Patients with simpler deformities, such as symmetric pectus excavatum, were adequately assessed with 2-D CT scans with calculation of the Haller Index, as well as the physical examination and patient symptoms, and may not require evaluation by 3-D reconstruction. However, the 3-D images of the bony deformities confer a better understanding to the patients and families about the specific deformity, and are generally greatly appreciated by them.

\section{Acknowledgments}

We thank: Paul Thomlinson, Ph.D. for providing the statistical analyses; Daniel Von Allmen, M.D., William Adamson, M.D., and John Van Aalst, M.D., for providing patients, images, and help with evaluation of their patients for this study. Stipend support to E.H.C. and A.N.C. in part by National Institutes of Health NIDDK grant \# T35DK007386. No financial support was supplied by Siemens Corporation. 


\section{REFERENCES}

1. Phillips JD, van Aalst JA. Jeune's syndrome (asphyxiating thoracic dystrophy): congenital and acquired. Semin. Pediatr. Surg. 2008 Aug; 17(3):167-172. [PubMed: 18582822]

2. Haller JA, Colombani PM, Humphries CT, Azizkhan RG, Loughlin GM. Chest wall constriction after too extensive and too early operations for pectus excavatum. Ann. Thorac. Surg. 1996 Jun; 61(6):1618-1624. discussion 1625. [PubMed: 8651758]

3. van Aalst JA, Phillips JD, Sadove AM. Pediatric chest wall and breast deformities. Plast. Reconstr. Surg. 2009 Jul; 124(1 Suppl):38e-49e.

4. Hebra A, Swoveland B, Egbert M, Tagge EP, Georgeson K, Othersen HB, et al. Outcome analysis of minimally invasive repair of pectus excavatum: review of 251 cases. J. Pediatr. Surg. 2000 Feb; 35(2):252-257. discussion 257-258. [PubMed: 10693675]

5. Nuss D, Kelly RE, Croitoru DP, Katz ME. A 10-year review of a minimally invasive technique for the correction of pectus excavatum. J. Pediatr. Surg. 1998 Apr; 33(4):545-552. [PubMed: 9574749]

6. Park HJ, Lee SY, Lee CS, Youm W, Lee KR. The Nuss procedure for pectus excavatum: evolution of techniques and early results on 322 patients. Ann. Thorac. Surg. 2004 Jan; 77(1):289-295. [PubMed: 14726081]

7. Engum S, Rescorla F, West K, Rouse T, Scherer LR, Grosfeld J. Is the grass greener? Early results of the Nuss procedure. J. Pediatr. Surg. 2000 Feb; 35(2):246-251. discussion 257-258. [PubMed: 10693674]

8. Fonkalsrud EW, Beanes S, Hebra A, Adamson W, Tagge E. Comparison of minimally invasive and modified Ravitch pectus excavatum repair. J. Pediatr. Surg. 2002 Mar; 37(3):413-417. [PubMed: 11877659]

9. Fonkalsrud EW, Dunn JC, Atkinson JB. Repair of pectus excavatum deformities: 30 years of experience with 375 patients. Ann. Surg. 2000 Mar; 231(3):443-448. [PubMed: 10714639]

10. Robicsek F, Fokin AA. How not to do it: restrictive thoracic dystrophy after pectus excavatum repair. Interact Cardiovasc Thorac Surg. 2004 Dec; 3(4):566-568. [PubMed: 17670312]

11. Weber TR. Further experience with the operative management of asphyxiating thoracic dystrophy after pectus repair. J. Pediatr. Surg. 2005 Jan; 40(1):170-173. [PubMed: 15868580]

12. Rattan AS, Laor T, Ryckman FC, Brody AS. Pectus excavatum imaging: enough but not too much. Pediatr Radiol. 2010 Feb; 40(2):168-172. [PubMed: 19813009]

13. Kim M, Lee KY, Park HJ, Kim H, Kang E, Oh YW, et al. Development of new cardiac deformity indexes for pectus excavatum on computed tomography: feasibility for pre- and post-operative evaluation. Yonsei Med. J. 2009 Jun 30; 50(3):385-390. [PubMed: 19568601]

14. Stephenson JT, Du Bois J. Compressive orthotic bracing in the treatment of pectus carinatum: the use of radiographic markers to predict success. J. Pediatr. Surg. 2008 Oct; 43(10):1776-1780. [PubMed: 18926206]

15. Nakagawa Y, Uemura S, Nakaoka T, Yano T, Tanaka N. Evaluation of the Nuss procedure using pre- and postoperative computed tomographic index. J. Pediatr. Surg. 2008 Mar; 43(3):518-521. [PubMed: 18358292]

16. Lai J, Wang C, Chang P. The measurement and designation of the pectus bar by computed tomography. J. Pediatr. Surg. 2009 Dec; 44(12):2287-2290. [PubMed: 20006011]

17. Egan JC, DuBois JJ, Morphy M, Samples TL, Lindell B. Compressive orthotics in the treatment of asymmetric pectus carinatum: a preliminary report with an objective radiographic marker. J. Pediatr. Surg. 2000 Aug; 35(8):1183-1186. [PubMed: 10945691]

18. Haller JA, Kramer SS, Lietman SA. Use of CT scans in selection of patients for pectus excavatum surgery: a preliminary report. J. Pediatr. Surg. 1987 Oct; 22(10):904-906. [PubMed: 3681619]

19. Lawson ML, Barnes-Eley M, Burke BL, Mitchell K, Katz ME, Dory CL, et al. Reliability of a standardized protocol to calculate cross-sectional chest area and severity indices to evaluate pectus excavatum. J. Pediatr. Surg. 2006 Jul; 41(7):1219-1225. [PubMed: 16818052]

20. Albes JM, Seemann MD, Heinemann MK, Ziemer G. Correction of anterior thoracic wall deformities: improved planning by means of 3D-spiral-computed tomography. Thorac Cardiovasc Surg. 2001 Feb; 49(1):41-44. [PubMed: 11243521] 
21. Pretorius ES, Haller JA, Fishman EK. Spiral CT with 3D reconstruction in children requiring reoperation for failure of chest wall growth after pectus excavatum surgery. Preliminary observations. Clin Imaging. 1998 Apr; 22(2):108-116. [PubMed: 9543588] 


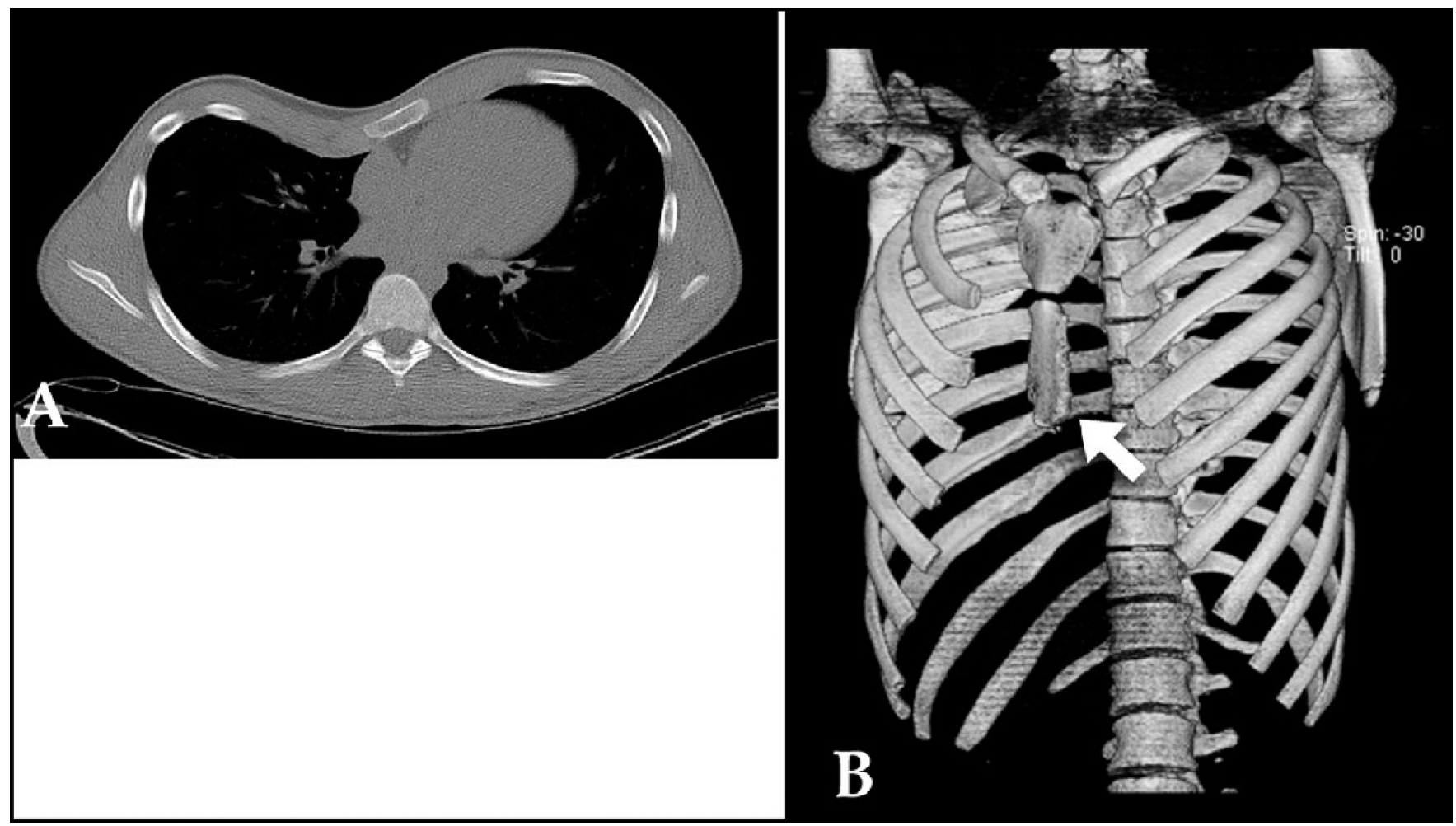

Figure 1.

A: 2-D CT cross section of 15 year old male with PE. B: SSD demonstrated ultrashort sternum (arrow), which is better clarified in 3-D than 2-D. Score: 2 

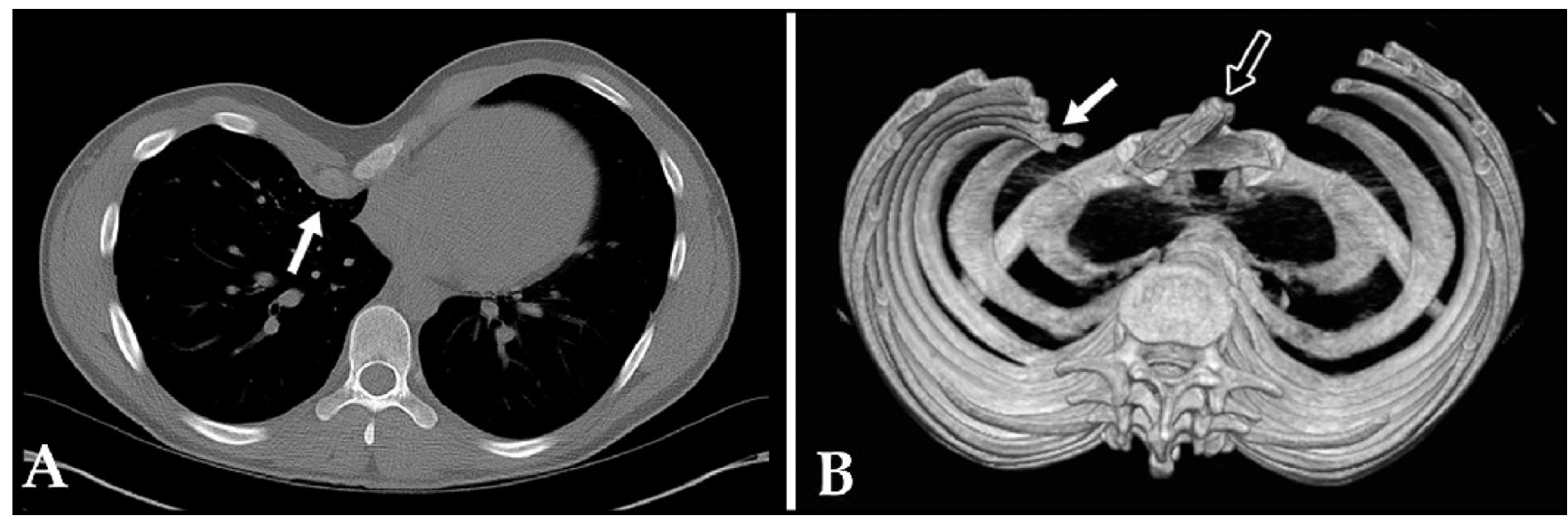

Figure 2.

A: 2-D CT cross-sectional image of 19 year old male with PE, showing asymmetry and inward protrusion of right para-sternal costal cartilages (arrow). B: SSD of same patient clarifying inward protrusion of right rib tips, as opposed to just cartilage (white arrow); SSD also helps clarify significant sterna rotation (black arrow). SSD influenced surgeon's decision about type of operation (Ravitch vs Nuss). Score: 2 


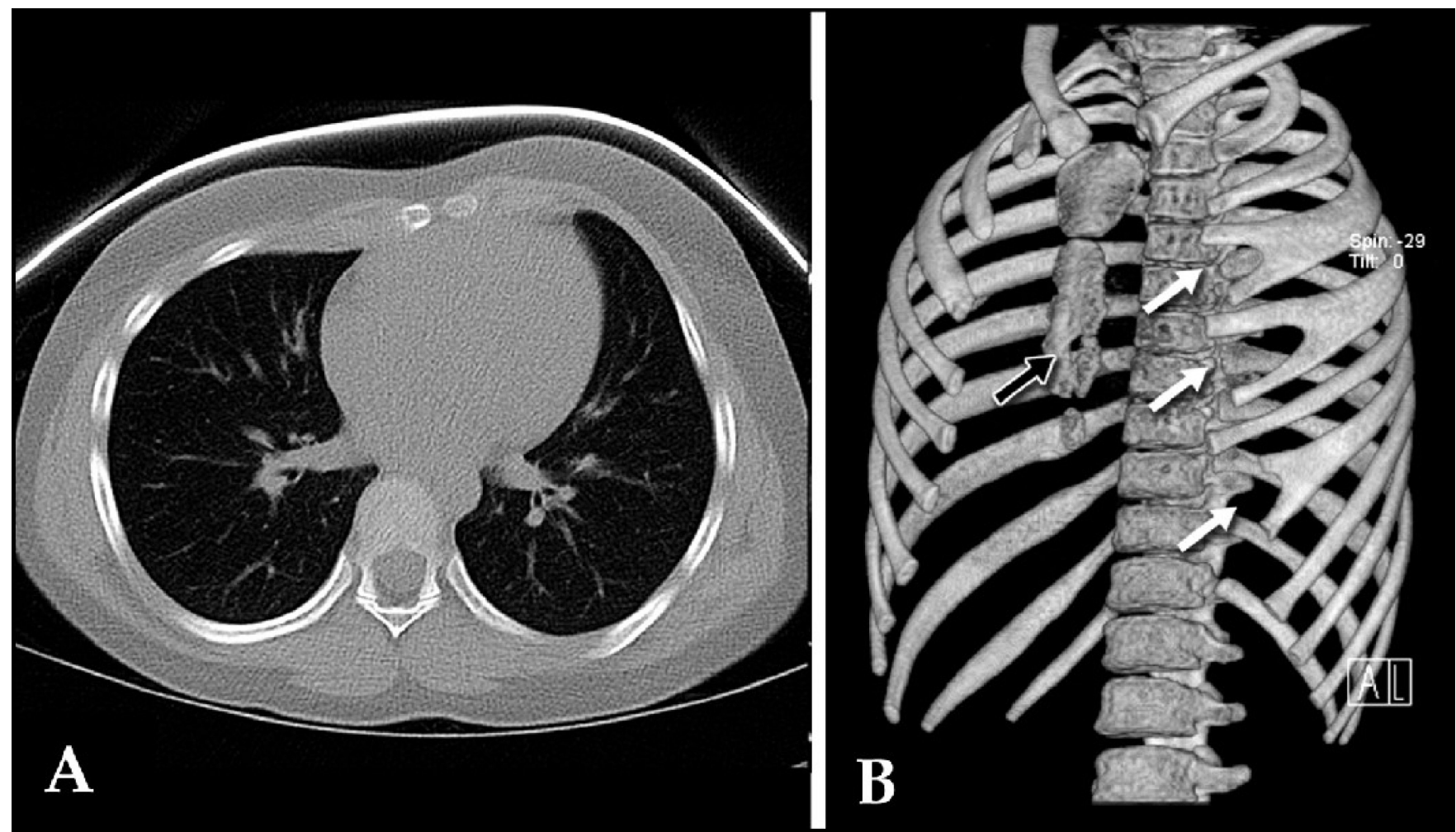

Figure 3.

A: 2-D CT cross-sectional image of 9 year old female with rapidly-progressive PC showing asymmetric protruding anterior chest wall. B: SSD shows bifid left ribs 3, 4, \& 6 (white arrows), which were poorly demonstrated with 2-D CT; also clarifies bony defect in sternum (black arrow). Score: 3 

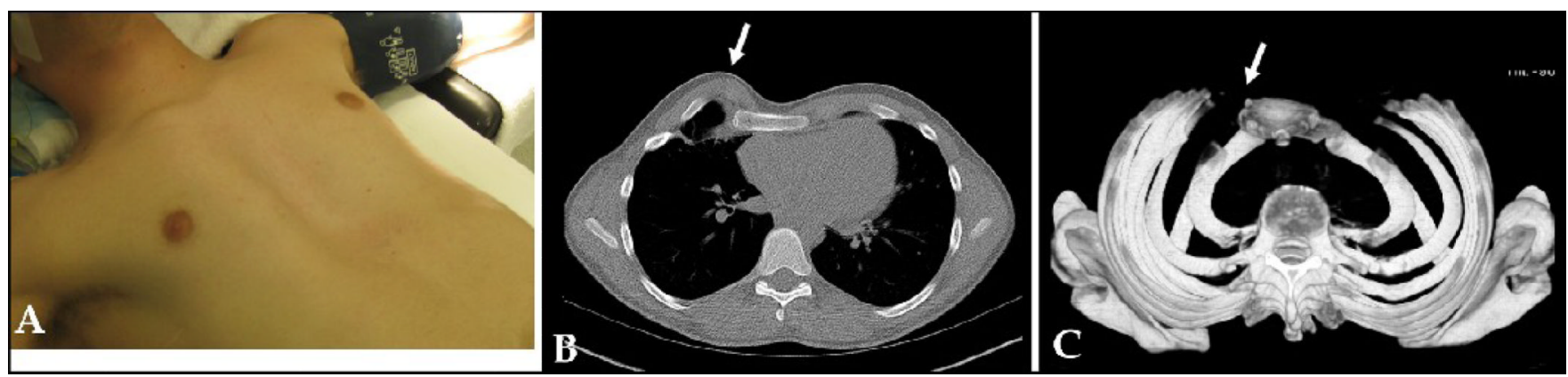

Figure 4.

A: 17 year old male with recurrent PE after previous Nuss repair. B: 2-D CT shows an ambiguous heaping deformity (arrow). C: SSD clarifies heaping deformity (arrow in similar position as in panel A), which is determined not to be from heterotopic calcifications, but primarily cartilaginous. SSD enables the surgeon to differentiate between bone and soft tissue. Patient then underwent a Ravitch repair. Score: 3 

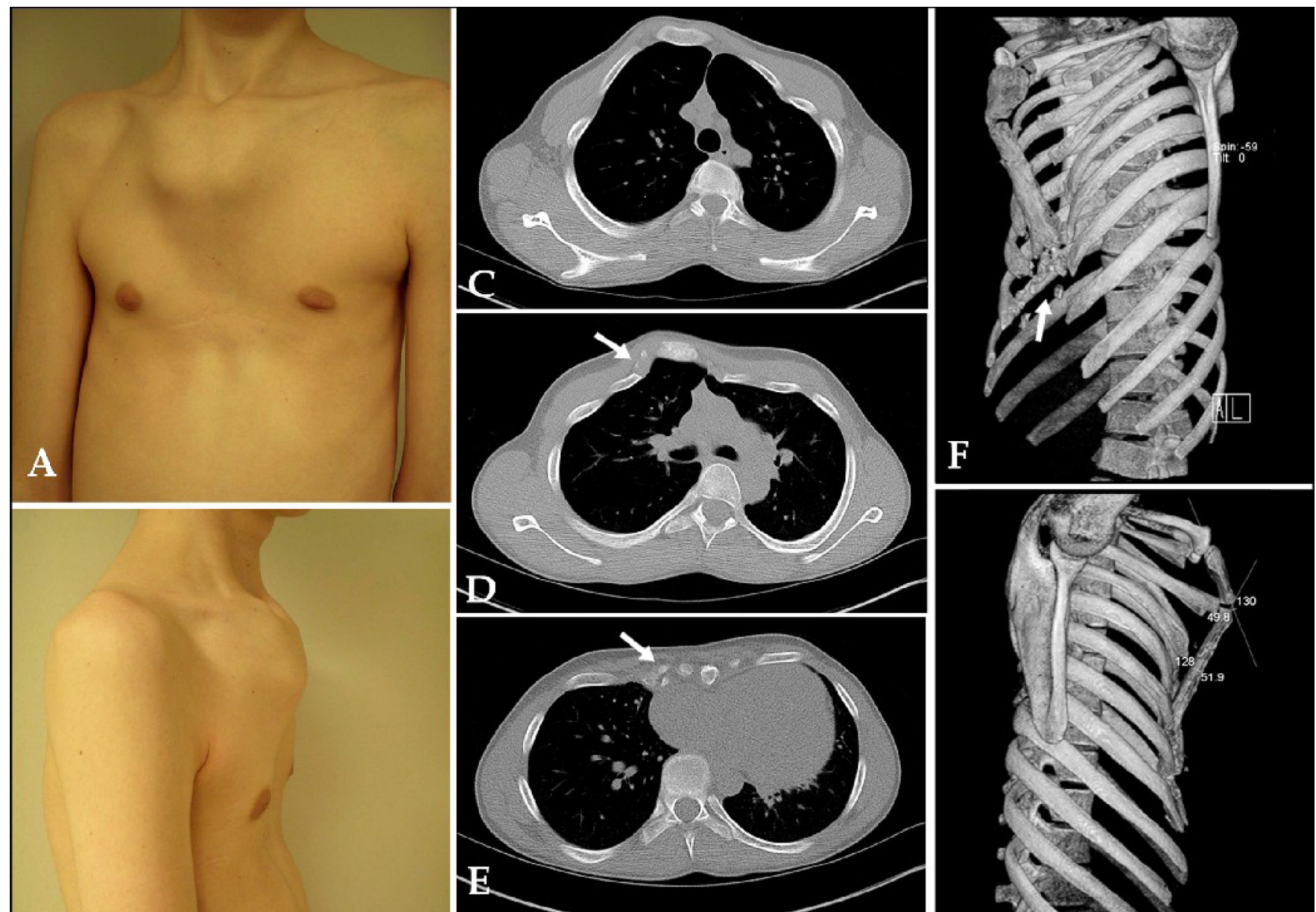

Figure 5.

A (frontal), B (lateral): 14 year old male with Marfan's, recurrent PE, and AATD after previous Ravitch procedure. C, D, E: 2-D CT cross sections showing heterotopic calcifications (arrows), bony abnormalities, and constricted chest cavity. F, G: SSD showing AATD and tiny chest, heterotopic calcifications (arrows), better view of inward rib curvature, and transverse sternal osteotomy from previous Ravitch. SSD influenced the surgeon to use 3 Nuss bars instead of 2 for this child's subsequent reconstruction. Score: 3 


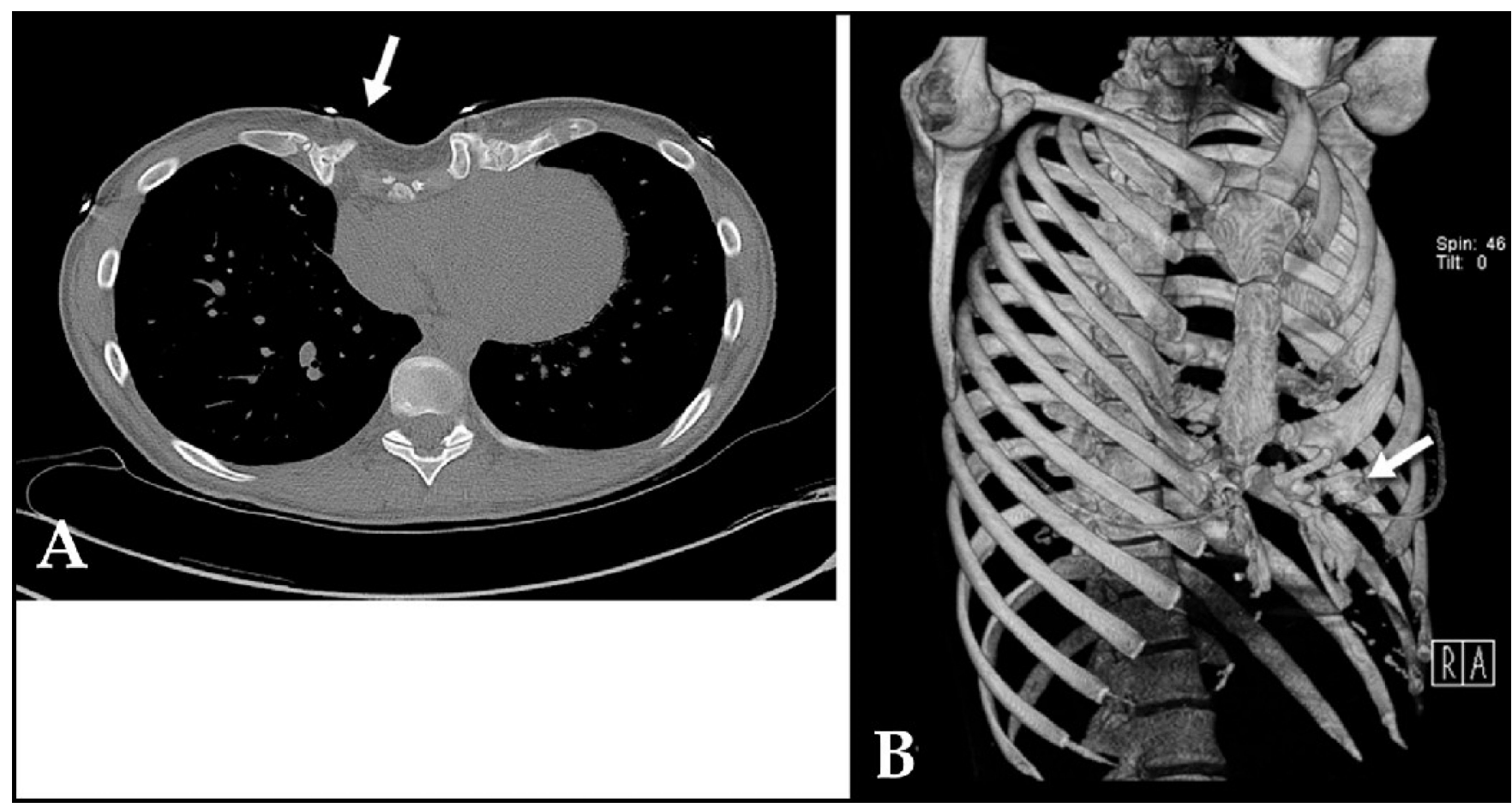

Figure 6.

A: 2-D CT of 21 year old female with recurrent PE after previous Ravitch repair showing heterotopic calcifications (arrow). B: SSD clarifies extent of heterotopic calcifications and bony deformities (arrow), influencing surgeon to perform a re-do Ravitch repair rather than Nuss. Score: 2 


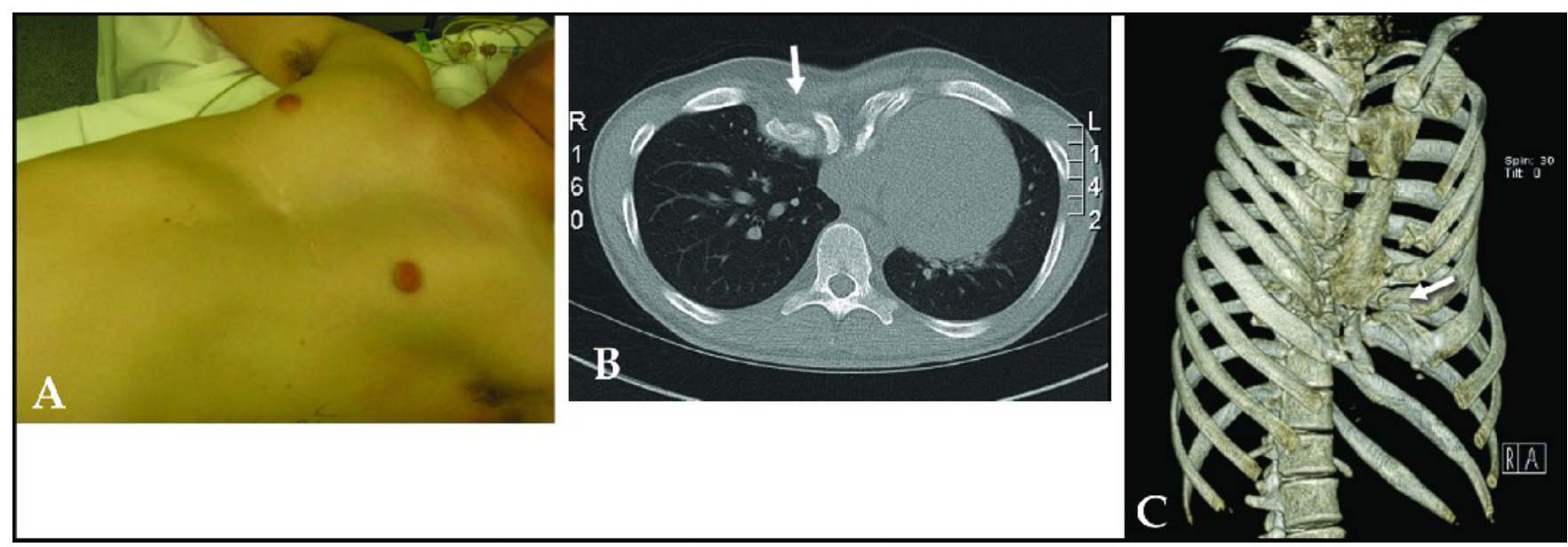

Figure 7.

A: 18 year old male with recurrent PE and bony abnormalities (arrow) after previous Ravitch repair. B: 2-D CT cross section showing AATD and bony abnormalities (arrow). C: SSD of same patient clarifying AATD, restricted chest cavity, and bony deformities. Score: 3 


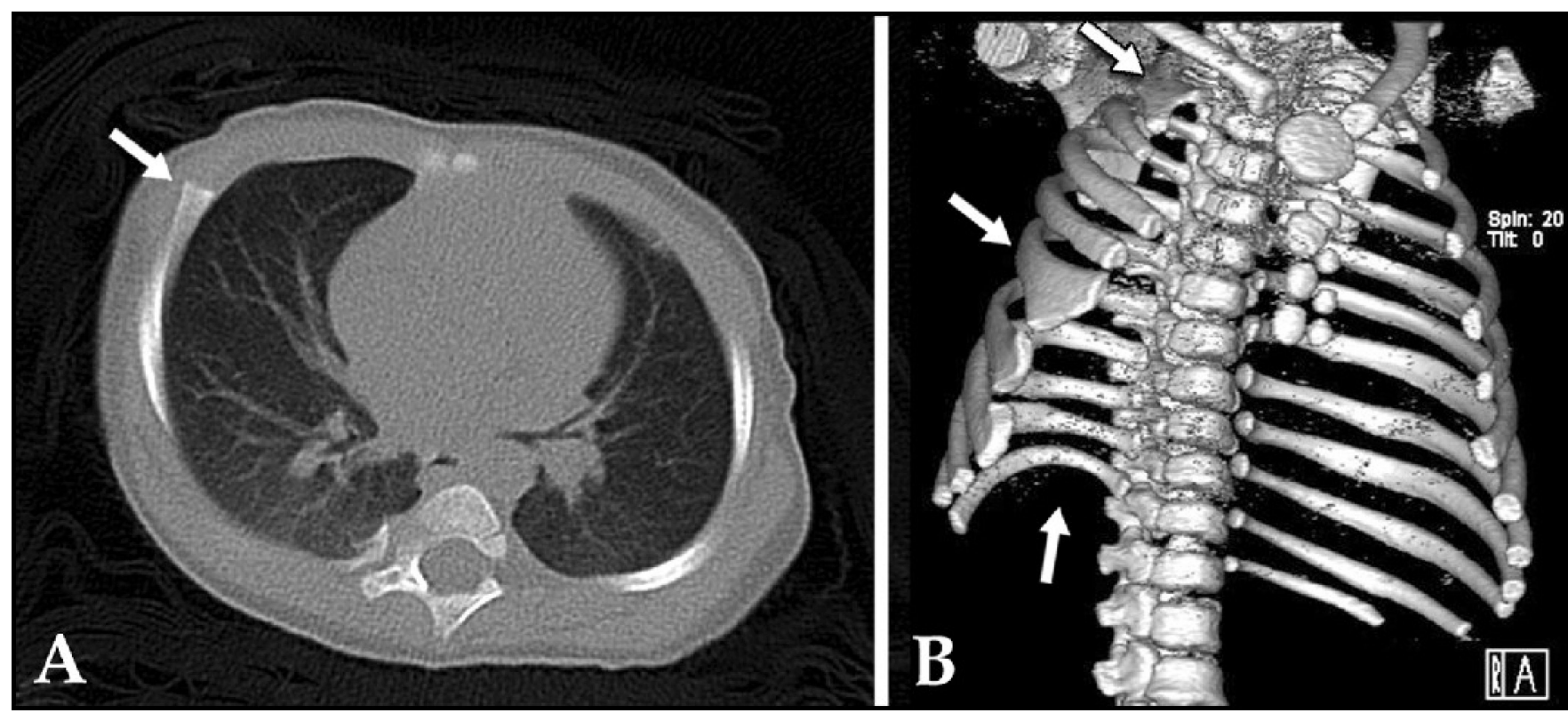

Figure 8.

A: 2-D CT cross-sectional image of 18 month old female, showing rib asymmetry and anterior protrusion (arrow) of the right parasternal cartilages (variant of pectus carinatum). B: SSD of same patient, showing bifid upper right rib (top arrow), multiple lower rib anomalies (middle arrow), absent right 12th rib (bottom arrow); also helps to clarify asymmetric/unusual sternal segmentation. Score: 3 
Table 1

Patient Diagnoses and Characteristics

\begin{tabular}{|l|l|l|}
\hline Patients & Number & $(\mathbf{\%})$ \\
\hline PE only & 53 & 65 \\
\hline PC only & 16 & 20 \\
\hline AATD \& PC or PE & 6 & 7 \\
\hline PC \& PE mix & 5 & 6 \\
\hline PS & 1 & 1 \\
\hline PS \& PC mix & 1 & 1 \\
\hline Previous Chest Repair & 11 & 13 \\
\hline Total & 82 & 100 \\
\hline
\end{tabular}

PE - pectus excavatum, PC - pectus carinatum, AATD - acquired asphyxiating thoracic dystrophy, PS - Poland's Syndrome. 


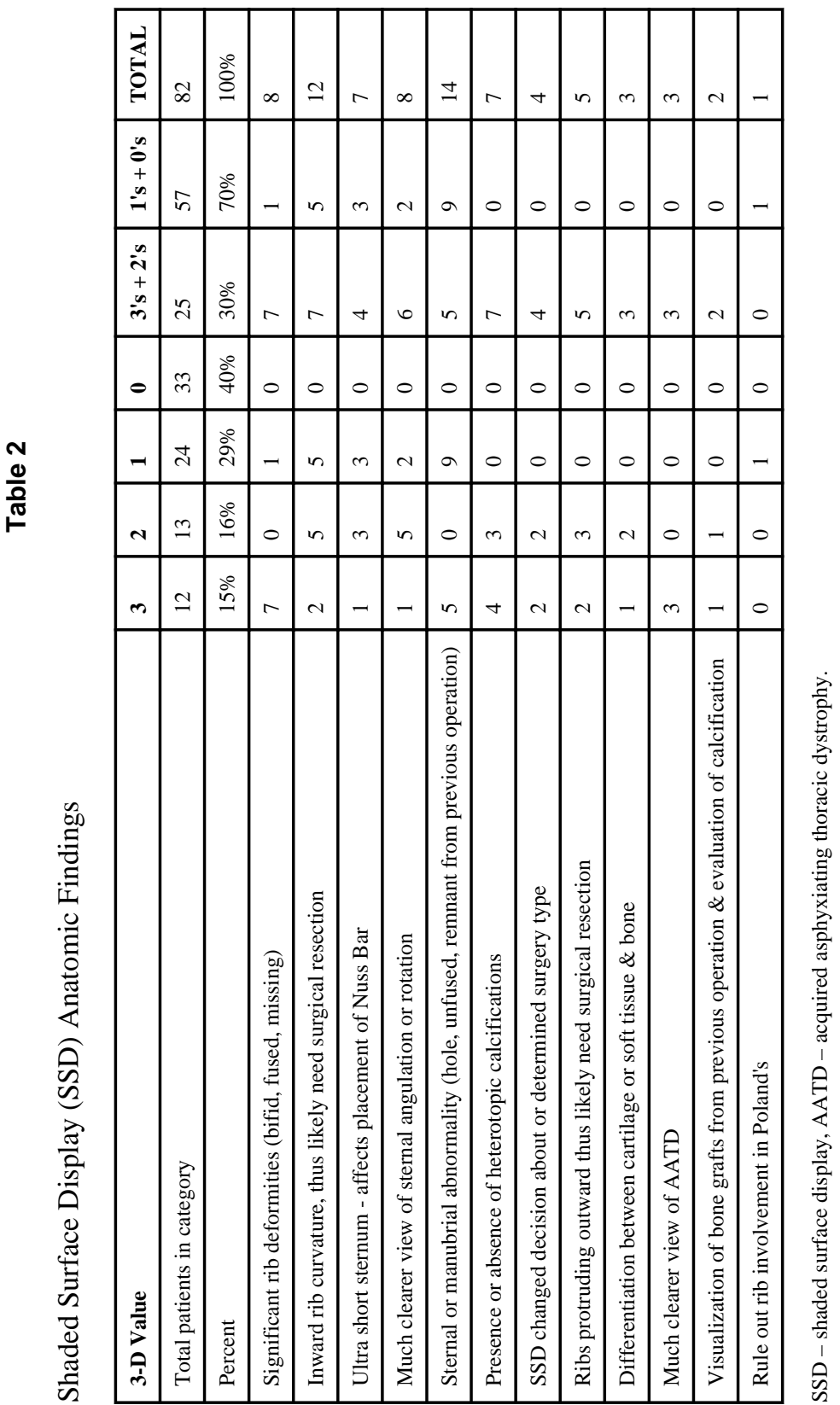

$\stackrel{n}{\sim}$

$n$
+
$m$
$m$

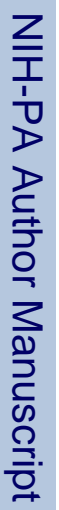

J Pediatr Surg. Author manuscript; available in PMC 2013 November 24. 
Table 3

Point Biserial Correlation Coefficient Analysis

\begin{tabular}{|l|l|l|}
\hline Parameter Tested & Prb & p-value \\
\hline Previous chest repair & 0.48 & $\unlhd 0.001^{*}$ \\
\hline AATD & 0.34 & $0.001^{*}$ \\
\hline PC & 0.27 & $0.008^{*}$ \\
\hline Female & 0.19 & $0.044^{*}$ \\
\hline Age & 0.22 & 0.102 \\
\hline PE & -0.18 & 0.202 \\
\hline
\end{tabular}

AATD - acquired asphyxiating thoracic dystrophy, PC - pectus carinatum, PE - pectus excavatum.

$\mathrm{p} \leq 0.05$ was considered statistically significant. 